Pacific Journal of Mathematic 


\section{NAOKI KIMURA}

1. Introduction. The first step in the study of idempotent semigroups has been made by David McLean [3] and is stated as follows.

Theorem 1. Let $S$ be an idempotent semigroup. Then there exist a semilattice $\Gamma$ and a disjoint family of rectangular subsemigroups of $S$ indexed by $\Gamma,\left\{S_{\gamma}: \gamma \in \Gamma\right\}$, such that

$$
S=\cup\left\{S_{\gamma}: \gamma \in \Gamma\right\}
$$

and

$$
S_{\gamma} S_{\delta} \subset S_{\gamma \delta}
$$$$
\text { for } r, \delta \in \Gamma \text {. }
$$

However, the structure of $S$ is not determined, in general, by knowing only the structures of $\Gamma$ and of all $S_{\gamma}$.

In this paper and the subsequent papers we shall study some special idempotent semigroups which are defined by some identities, where the decomposition theorem above plays an important role. This paper will be chiefly concerned with the study of regular idempotent semigroups (for the definition see below), which can be considered as a quite general class of idempotent semigroups. Also characterizations of identities for some special idempotent semigroups are obtained.

2. Rectangular bands. An idempotent semigroup or band [1] is a semigroup which satisfies the identity $a^{2}=a$.

A semigroup satisfying the identity

$$
a b a=a \quad(a b=a, b a=a)
$$

is called rectangular (left singular, right singular). These semigroups are all idempotent. And a left (right) singular semigroup is rectangular. Conversely we have the following

Lemma 1. A rectangular semigroup is the direct product of a left singular semigroup and a right singular semigroup. Moreover this factorization is unique up to isomorphism.

Proof. Let $S$ be a rectangular semigroup. Then since

Received December 20, 1957, and in revised form February 26, 1958. This work was partially supported by the National Science foundation, U.S. A. An abstract of this paper has appeared in Proc. Jap. Acad., 33 (1957) p. 642. 


$$
x S \supset x(y S)=(x y) S \supset(x y)(x S)=(x y x) S=x S,
$$

we have

$$
\left\{\begin{array}{l}
x y S=x S . \text { Dually } \\
S x y=S y .
\end{array}\right.
$$

Therefore we have also

$$
\left\{\begin{array}{l}
(x S)(y S)=(x S)(y x S)=(x S y x) S=x S . \quad \text { Dually }, \\
(S x)(S y)=S y .
\end{array}\right.
$$

Let $A(B)$ be the set of all subsets of $S$ of the form $x S(S x)$. Then $A(B)$ forms a left (right) singular semigroup, with respect to the usual multiplication induced by that of $S$, on account of (2).

Let $p: S \rightarrow A(q: S \rightarrow B)$ be the mapping defined by

$$
p(x)=x S \quad(q(x)=S x) .
$$

Then by (1) and (2), $p$ and $q$ are onto homomorphisms.

Let $r: S \rightarrow A \times B$ be the mapping defined by

$$
r(x)=(p(x), q(x)) \text {. }
$$

Then $r$ is a homomorphism. Take any element of $A \times B$, say $(x S, S y)$. Then $r(x y)=(x y S, S x y)=(x S, S y)$, by (1). Thus $r$ is onto. On the other hand, if $r(z)=(x S, S y)$, then $z S=x S$ and $S z=S y$. Therefore by rectangularity we have

$$
x y=(x S x)(y S y)=(z S x)(y S z)=z(S x y S) z=z .
$$

Thus $r$ is an isomorphism between $S$ and $A \times B$, where $A(B)$ is left (right) singular.

Let $r^{\prime}: S \rightarrow A^{\prime} \times B^{\prime}$ be an isomorphism, where $A^{\prime}\left(B^{\prime}\right)$ is left (right) singular. Define $p^{\prime}: S \rightarrow A^{\prime}$ and $q^{\prime}: S \rightarrow B^{\prime}$ by $r^{\prime}(x)=\left(p^{\prime}(x), q^{\prime}(x)\right)$, then they are onto homomorphisms.

If $p(x)=p(y)$, that is $x S=y S$, then

$$
p^{\prime}(x S)=p^{\prime}(x) p^{\prime}(S)=p^{\prime}(x) \quad \text { and } \quad p^{\prime}(y S)=p^{\prime}(y) .
$$

Therefore $p^{\prime}(x)=p^{\prime}(y)$. Thus we have an onto homomorphism $f: A \rightarrow A^{\prime}$ $\left(g: B \rightarrow B^{\prime}\right)$ such that $p^{\prime}=f p\left(q^{\prime}=g q\right)$.

Now $f(g)$ must be one-to-one. For, let $x S \neq y S, f(x S)=f(y S)$. Then $x y S=x S \neq y S$, therefore $x y \neq y$. But

$$
\begin{gathered}
p^{\prime}(x y)=f p(x y)=f(x y S)=f(x S)=f(y S)=f p(y)=p^{\prime}(y), \\
q^{\prime}(x y)=g q(x y)=g(S x y)=g(S y)=g q(y)=q^{\prime}(y) .
\end{gathered}
$$

Therefore $r^{\prime}(x y)=r^{\prime}(y)$, which contradicts the assumption that $r^{\prime}$ is an 
isomorphism. Thus $f$ and $g$ must be isomorphisms.

This ends the proof of Lemma 1 .

REMARK 1. The above defined $A(B)$ is the set of all minimal right (left) ideals of $S$.

LEMmA 2. A band is rectangular if and only if it satisfies the identity $a b c=a c$.

Proof. (1) Sufficiency. If a band $S$ satisfies the above identity, then simply put $c=a$, which proves that $S$ is rectangular.

(2) Necessity. Assume that $S$ is a rectangular band, then $a(b c) a=$ $a$. Therefore $a b c=a b(c a c)=(a b c a) c=a c$, which proves the above identity .

REMARK 2. Now we have established the equivalence between two identities, $a b a=a$ and $a b c=a c$, on idempotent semigroups. Thus either one of them can define rectangularity.

Also each one of the following identities on bands is equivalent to rectangularity :

$$
\begin{gathered}
a x_{1} x_{2} \cdots x_{n} a=a \\
a x_{1} \cdots x_{i-1} a x_{i} \cdots x_{j-1} a x_{j} \cdots \cdots \cdots x_{n} a=a \\
(1<i<j<\cdots<n), \\
a x_{1} x_{2} \cdots x_{n} b=a b \\
a x_{1} \cdots x_{i-1} c_{1} x_{i} \cdots x_{j-1} c_{2} x_{j} \cdots \cdots \cdots x_{n} b=a b,
\end{gathered}
$$

where $c_{k}$ is either $a$ or $b$ for each $k(1<i<j<\cdots<n)$,

$$
\begin{aligned}
a x_{1} x_{2} \cdots x_{n} b=a x_{i_{1}} x_{i_{2}} \cdots x_{i_{r}} b & \\
& \left(1 \leqq i_{1}<i_{2}<\cdots<i_{r} \leqq n, r<n\right) .
\end{aligned}
$$

These facts raise the problem of determining the conditions for identities to be equivalent to rectangularity. It will be discussed in $\S 5$ below, and there we will find that the equivalence of the above identities with rectangularity is merely a special case of Theorem 6 .

REMARK 3. If we consider the two identities, $a b a=a$ and $a b c=a c$, for general semigroups, then they are not equivalent. The former defines a rectangular band, but the latter defines a little wider class of semigroups which contains rectangular bands.

However we have the following Lemma 3.

A semigroup $S$ is called total if every element of $S$ can be written as the product of two elements of $S$, that is $S^{2}=S$. 
Lemma 3. A total semigroup is rectangular if and only if it satisfies the identity $a b c=a c$.

Proof. (1) Sufficiency Let $S$ be total. Assume the identity $a b c=$ ac. Pick $a \in S$, then $a=x y$ for some elements $x, y$. Then

$$
a^{2}=(x y)^{2}=(x y)(x y)=x(y x) y=x y=a .
$$

Thus $S$ is a band. Therefore by Lemma $2, S$ must be rectangular.

(2) Necessity. Obvious, because any rectangular semigroup satisfies the given identity by Lemma 2 . This ends the proof of Lemma 3.

Let $S$ be a semigroup which satisfies the identity $a b c=a c$. Consider the mapping $f: S \rightarrow S$ defined by $f(x)=x^{2}$. Then $f$ is a homomorphism of $S$ into $S$, because

$$
f(x y)=(x y)^{2}=x(y x) y=x y=x(x y) y=x^{2} y^{2}=f(x) f(y) .
$$

Let $R$ be the image of $S$ under $f$ :

$$
R=f(s)=\left\{x^{2}: x \in S\right\} .
$$

Then obviously $R^{2} \subset R \subset S^{2}$. Conversely, every element of $S^{2}$ is idempotent, because $(x y)^{2}=x(y x) y=x y$. Therefore we have $R^{2}=R=S^{2}$. Now since $R$ is total, $R$ is rectangular by Lemma 3 .

Hence, defining $S_{r}$ by $S_{r}=\left\{x: x \in S, x^{2}=r\right\}, S$ is decomposed in the following way :

$$
S=\cup\left\{S_{r}: r \in R\right\} \text {, where } S, S_{t}=\{r t\} .
$$

For, if $x \in S_{r}, y \in S_{t}$, then $x^{2}=r, y^{2}=t$ and so $x y=x^{2} y^{2}=r t$. Thus we have the following

THEOREM 2. Let $S$ be a semigroup satisfying the identity abc=ac. Then there exists a rectangular subsemigroup $R$ of $S$ and a partition of $S$ with $R$ as its index set, such that

$$
S=\cup\left\{S_{r}: r \in R\right\},
$$

where

$$
\begin{aligned}
& S_{r} \cap S_{t}=\square, \text { the null set, } \quad \text { if } r \neq t, \\
& r \in S_{r}
\end{aligned}
$$

and

$$
S_{r} S_{t}=\{r t\}
$$

Thus the "if" part of Lemma 3 is a special case of this Theorem. 
3. The structure of one-sided regular bands. A band is called (1) left regular, (2) right regular or (3) regular if it satisfies the identity :

$$
\begin{aligned}
& a b a=a b, \\
& a b a=b a
\end{aligned}
$$

or

$$
a b a c a=a b c a,
$$

respectively.

Then the following lemmas are obvious by these definitions.

Lemma 4. A left (right) regular band is regular.

Lemma 5. The direct product of (left, right) regular bands is also (left, right) regular.

LEMma 6. Any subsemigroup of a (left, right) regular band is also (left, right) regular.

Lemma 7. A left (right) singular band is left (right) regular.

Lemma 8. A rectangular band is regular.

Lemma 9. A band is left (right) singular if and only if it is both left (right) regular and rectangular.

LEMMA 10. A band is commutative if and only if it is both left and right regular.

For a total semigroup we have the following.

LEMMA 11. A total semigroup is a left (right) regular band if and only if it satisfies the identity $a b a=a b(a b a=b a)$.

Proof. The necessity is trivial. So it is sufficient to prove the idempotence from the above identity

Let $S$ be a total semigroup, that is $S^{2}=S$. Then any element $x \in S$ can be written as the product of two elements of $S$, say, $x=a b$ for some $a, b \in S$. Therefore

$$
x^{2}=(a b)^{2}=a(b a b)=a(b a)=a b a=a b=x .
$$

Thus we have $x^{2}=x$, or $S$ is idempotent. 
Let $S$ be a band. Then by Theorem 1 there exist a semilattice $\Gamma$ and a disjoint family of rectangular subsemigroups of $S$ indexed by $\Gamma$, $\left\{S_{\gamma}: \gamma \in \Gamma\right\}$, such that

$$
S=\bigcup\left\{S_{\gamma}: \gamma \in \Gamma\right\}
$$

and

$$
S_{\gamma} S_{\delta} \subset S_{\gamma \delta} \quad \text { for } \quad \gamma, \delta \in \Gamma
$$

(See McLean [3, p. 111], also see A. H. Clifford [1, p. 501]).

Furthermore $\Gamma$ is determined uniquely up to isomorphism, and accordingly so is $S_{\gamma}$.

We call $\Gamma$ the structure semilattice, and $S_{\gamma}$ the $(\gamma$-)kernel. A homomorphism $p: S \rightarrow \Gamma$ defined by $p\left(S_{\gamma}\right)=\gamma$ is called natural. Also in this case we write $S \sim \sum\left\{S_{\gamma}: \gamma \in \Gamma\right\}$, and call it the structure decomposition of $S$.

Then we have the following corollaries to Theorem 1 .

CoRollary 1. Each kernel $S_{\gamma}$ is a maximal rectangular subsemigroup of $S$. Moreover any rectangular subsemigroup of $S$ is contained in one and only one kernel.

Proof. Let $S \sim \sum\left\{S_{\gamma}: \gamma \in \Gamma\right\}$ be the structure decomposition of $S$ and let $p: S \rightarrow \Gamma$ be natural. If $R$ is a rectangular subsemigroup of $S$, then $p(R)$ is also a rectangular subsemigroup of $\Gamma$. Since $\Gamma$ is a semilattice, $p(R)$ is reduced to a single element, say $\gamma=p(R)$, and according $R \subset p^{-1}(\gamma)=S_{\gamma}$. Namely $R$ is contained in one and only one $S_{\gamma}$ since the $S_{\gamma}$ 's are disjoint. On the other hand $S_{\gamma}$ is rectangular for each $\gamma \in \Gamma$. Therefore each kernel $S_{\gamma}$ is a maximal rectangular subsemigroup of $S$.

Corollary 2. For any (onto) homomorphism $q: S \rightarrow \Delta$, where $\Delta$ is a semilattice, there exists a unique (onto) homomorphism $f: \Gamma \rightarrow \Delta$, such that $q=f p$, where $p: S \rightarrow \Gamma$ is natural.

Proof. Since $q\left(S_{\gamma}\right)$ is rectangular, it must be a single element in $\Delta$. Now we have a mapping $f: \Gamma \rightarrow \Delta$ defined by $f(\gamma)=q\left(S_{\gamma}\right)$. Then it is easy to see that $q=f p$.

CoRollary 3. Let $q: S \rightarrow \Delta$ be an onto homomorphism, where $\Delta$ is a semilattice. If $q^{-1}(\delta)$ is rectangular for all $\delta \in \Delta$, then the mapping $f$ defined above is an isomorphism. More precisely, we can consider $\Delta$ as the structure semilattice of $S, q^{-1}(\delta)$ as the $\delta$-kernel and $q$ as the natural homomorphism, that is $S \sim \sum\left\{q^{-1}(\delta): \delta \in \Delta\right\}$.

Proof. Since $q^{-1}(\delta)$ is rectangular, it is contained in $S_{\gamma}$ for some $\gamma$ by Corollary 1 above. Now we have 


$$
\gamma=p\left(S_{\gamma}\right) \supset p q^{-1}(\delta)=p(f p)^{-1}(\delta)=p p^{-1} f^{-1}(\delta)=f^{-1}(\delta) .
$$

Therefore $f$ must be one-to-one.

THEOREM 3. A band is left (right) regular, if and only if its kernels are all left (right) singular (Naoki Kimura [2, p. 117]).

Proof. Let $S \sim \sum\left\{S_{\gamma}: \gamma \in \Gamma\right\}$ be the structure decomposition of a band $S$.

(1) Let $S$ be left regular. Then each $\gamma$-kernel $S_{\gamma}$ of $S$ is rectangular. Also it is left regular by Lemma 6 .

Therefore $S_{\gamma}$ must be left singular by Lemma 9 .

(2) Let every kernel of $S$ be left singular. Let $a \in S_{\alpha}, b \in S_{\beta}$. Then $a b, b a \in S_{\alpha \beta}=S_{\beta \alpha}$. Thus, by the left singularity of $S_{\alpha \beta}$, we have $a b a=a b^{2} a=(a b)(b a)=a b$, which proves that $S$ is left regular.

4. The structure of regular bands. Let $\Gamma$ be a semilattice. Let $A$ and $B$ be bands having $\Gamma$ as their structure semilattice. Let $A \sim \sum\left\{A_{\gamma}\right.$ : $\gamma \in \Gamma\}, B \sim \sum\left\{B_{\gamma}: \gamma \in \Gamma\right\}$ be their structure decompositions.

Form the direct product $D=A \times B$. Then $C_{\gamma}=A_{\gamma} \times B_{\gamma}$ can be considered as a rectangular subsemigroup of $D$. Also $C=\cup\left\{C_{\gamma}: \gamma \in \Gamma\right\}$ is a subsemigroup of $D$. Moreover the structure decomposition of $C$ is $C \sim$ $\sum\left\{C_{\gamma}: \gamma \in \Gamma\right\}$.

Let $p: A \rightarrow \Gamma, q: B \rightarrow \Gamma$ be the natural homomorphisms. Then

$$
C=\{(x, y): x \in A, y \in B, p(x)=q(y)\},
$$

and $r: C \rightarrow \Gamma$ defined by $r(x, y)=p(x)=q(y)$ is the natural homomorphism. We call $C$ the spined product of $A$ and $B$ with respect to $\Gamma$. Note that this product depends not only on $A, B$ and $\Gamma$ but also on the natural homomorphism $p$ and $q$ [2, p. 28].

LEMMA 12. The spined product of a left regular band and a right regular band is regular.

Proof. Since the spined product of $A$ and $B$ is a subsemigroup of the direct product of $A$ and $B$, we have the lemma by Lemmas 4,5 and 6.

Now we shall prove the converse of this lemma which plays an essential part in the structure theorem of regular bands.

Lemma 13. Let $S \sim \sum\left\{S_{\gamma}: \gamma \in \Gamma\right\}$ be a regular band. Then there exist a left regular band $A \sim \sum\left\{A_{\gamma}: \gamma \in \Gamma\right\}$ and a right regular band $B \sim$ $\sum\left\{B_{\gamma}: \gamma \in \Gamma\right\}$, both of which have the same structure semilattice $\Gamma$, such 
that $\boldsymbol{S}$ is isomorphic to the spined product of $A$ and $B$ with respect to $\Gamma$.

Proof. Let $\boldsymbol{S} \sim \sum\left\{S_{\gamma}: \gamma \in \Gamma\right\}$ be a regular band. Since each $\gamma$-kernel $S_{\gamma}$ is rectangular we can assume that $S_{\gamma}=A_{\gamma} \times B_{\gamma}$, where $A_{\gamma}$ is left singular and $B_{\gamma}$ is right singular. Let

$$
A=\cup\left\{A_{\gamma}: \gamma \in \Gamma\right\}, B=\cup\left\{B_{\gamma}: \gamma \in \Gamma\right\}, T=A \times B .
$$

Then $S$ can be identified as a subset of $T$. We shall prove that $A$ and $B$ can be considered as idempotent semigroups. Let

$$
a \in A_{\alpha}, c \in A_{\beta}, b, b^{\prime} \in B_{\alpha}, d, d^{\prime} \in B_{\beta} .
$$

Then $(a, b),\left(a, b^{\prime}\right) \in \boldsymbol{S}_{\alpha},(c, d),\left(c, d^{\prime}\right) \in S_{\beta}$. Put $(e, f)=(a, b)(c, d),\left(e^{\prime}, f^{\prime}\right)=$ $\left(a, b^{\prime}\right)\left(c, d^{\prime}\right)$. Then both $(e, f)$ and $\left(e^{\prime}, f^{\prime}\right)$ belong to $S_{\alpha \beta}$.

Since $A_{\alpha \beta}$ is left singular and $B_{\alpha \beta}$ is right singular, we have

$$
(e, f)\left(e^{\prime}, f^{\prime}\right)=\left(e, f^{\prime}\right) \text {. }
$$

On the other hand we have

$$
\begin{aligned}
(e, f)\left(e^{\prime}, f^{\prime}\right)=(a, b)(c, d)\left(a, b^{\prime}\right)\left(c, d^{\prime}\right) & \\
= & \left(a, b^{\prime} b\right)\left(c, d^{\prime} d\right)\left(a, b b^{\prime}\right)(c, d) \quad\left(\text { by right singularity of } B_{a} \text { and } B_{\beta}\right) \\
= & \left(a, b^{\prime}\right)(a, b)\left(c, d^{\prime}\right)(c, d)(a, b)\left(a, b^{\prime}\right)\left(c, d^{\prime}\right) \\
= & \left(a, b^{\prime}\right)(a, b)\left(a, b^{\prime}\right)\left(c, d^{\prime}\right)(a, b)(c, d)(a, b)\left(a, b^{\prime}\right)\left(c, d^{\prime}\right) \\
& =\left(a, b^{\prime} b b^{\prime}\right)\left(c, d^{\prime}\right)(a, b)(c, d)\left(a, b b^{\prime}\right)\left(c, d^{\prime}\right) \\
= & \left(a, b^{\prime}\right)\left(c, d^{\prime}\right)(a, b)(c, d)\left(a, b^{\prime}\right)\left(c, d^{\prime}\right) \\
= & \left(e^{\prime}, f^{\prime}\right)(e, f)\left(e^{\prime}, f^{\prime}\right) \\
= & \left(e^{\prime}, f^{\prime}\right) .
\end{aligned}
$$

Hence

$$
\left(e, f^{\prime}\right)=\left(e^{\prime}, f^{\prime}\right) \text { or } \quad e=e^{\prime} .
$$

Thus $e$ is determined by $a$ and $c$ only, and does not depend on $b$ or d. Similarly, $f$ is also determined by $b$ and $d$ only.

Now we can define $m: A \times A \rightarrow A, n: B \times B \rightarrow B$ by

$$
(m(a, c), n(b, d))=(a, b)(c, d)=(e, f) .
$$

Thus $A$ and $\mathrm{B}$ become multiplicative systems where $m$ and $n$ are multiplications on them, and $A_{\gamma}$ and $B_{\gamma}$ are subsystems which are a left singular band and a right singular band, respectively. Also $T=A \times B$ is a multiplicative system. 
Consider the projections $p: T \rightarrow A$ defined by $p(a, b)=a$ and $q: T \rightarrow$ $B$ defined by $q(a, b)=b$. They are homomorphisms. Therefore the mappings $p$ and $q$ with their domain restricted to $S \subset T$ are also homomorphisms, and their images are $A=p(S)$ and $B=q(S)$. Since homomorphisms preserve any relation defined by identities, as a result, associativity and idempotency hold in both $A$ and $B$, because $S$ is a band. Thus both $A$ and $B$ are bands.

Since $A_{\gamma}$ is left singular, and $B_{\gamma}$ is right singular, they are rectangular, and since $\Gamma$ is a semilattice,

$$
A \sim \sum\left\{A_{\gamma}: \gamma \in \Gamma\right\}, \quad B \sim \sum\left\{B_{\gamma}: \gamma \in \Gamma\right\}
$$

become the structure decompositions of $A$ and $B$, by Corollary 3 to Theorem 1.

Thus there exist a left regular band $A$ and a right regular band $B$ such that $S$ is the spined product of $A$ and $B$ with respect to $\Gamma$.

Lemmas 12 and 13 prove the following

THEOREM 4. A band is regular if and only if it is the spined product of a left regular band and a right regular band. ${ }^{1}$

CoRollary 1. Any regular band is imbedded into the direct product of a left regular band and a right regular band.

Proof. Immediately from Theorem 4.

Corollary 2. Let $S$ be the spined product of $A$ and $B$ with respect to $\Gamma$ and let $T$ be the spined product of. $C$ and $D$ with respect to $\Delta$, where $A \sim \sum\left\{A_{\gamma}: \gamma \in \Gamma\right\}$ and $C \sim \sum\left\{C_{\delta}: \delta \in \Delta\right\}$ are left regular, and $B \sim \sum\left\{B_{\gamma}\right.$ : $\gamma \in \Gamma\}$ and $D \sim \sum\left\{D_{\delta}: \delta \in \Delta\right\}$ are right regular.

Let $k: S \rightarrow T$ be a homomorphism, then there exist a homomorphism $h: \Gamma \rightarrow \Delta$ and homomorphisms $f: A \rightarrow C$ and $g: B \rightarrow D$ satisfying (1) $k(a, b)=(f(a), g(b))$ and $(2) h p=r f$ and $h q=s g$, that is the diagram

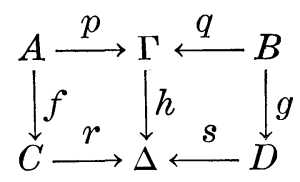

is analytic, where $p, q, r$ and $s$ are the natural homomorphisms.

Proof. Let $u: S \rightarrow \Gamma, v: T \rightarrow \Delta$ be the natural homomorphisms. Then since $v k: S \rightarrow \Delta$ is a homomorphism, by Corollary 2 to Theorem 1 , there exists a unique homomorphism $h: \Gamma \rightarrow \Delta$ such that $v k=h u$.

Therefore $v\left(k\left(S_{\gamma}\right)\right)=h u\left(S_{\gamma}\right)=h(\gamma)$, and so $k\left(S_{\gamma}\right) \subset v^{-1}(\delta)=T_{\delta}$, where $\delta=$ $h(\gamma)$. Now the homomorphism $k_{\gamma}: S_{\gamma} \rightarrow T_{\delta}$ defines uniquely homomorphisms $f_{\gamma}: A_{\gamma} \rightarrow C_{\delta}$ and $g_{\gamma}: B_{\gamma} \rightarrow D_{\delta}$ such that $k_{\gamma}(a, b)=\left(f_{\gamma}(a), g_{\gamma}(b)\right)$,

${ }_{1}$ Miyuki Yamada has obtained this theorem also, according to a recent communication from him to the author. 
where $k_{\gamma}$ is the homomorphism $k$ with its domain restricted to $S_{\gamma}$.

Since $A$ and $B$ are the union of $A_{\gamma}$ and $B_{\gamma}$ for $\gamma \in \Gamma, f_{\gamma}$ and $g_{\gamma}$ determined uniquely mappings $f: A \rightarrow C$ and $g: B \rightarrow D$, such that

$$
f(a)=f_{\gamma}(a) \text { if } \quad a \in A_{\gamma},
$$

and

$$
g(b)=g_{\gamma}(b) \quad \text { if } \quad b \in B_{\gamma} .
$$

Then it is obvious that $k(a, b)=(f(a), g(b))$. Therefore if $(a, b) \in S$, $\left(a^{\prime}, b^{\prime}\right) \in S$, then we have

$$
\begin{aligned}
\left(f\left(a a^{\prime}\right), g\left(b b^{\prime}\right)\right) & =k\left(a a^{\prime}, b b^{\prime}\right)=k\left((a, b)\left(a^{\prime}, b^{\prime}\right)\right)=k(a, b) k\left(a^{\prime}, b^{\prime}\right) \\
& =(f(a), g(b))\left(f\left(a^{\prime}\right), g\left(b^{\prime}\right)\right)=\left(f(a) f\left(a^{\prime}\right), g(b) g\left(b^{\prime}\right)\right),
\end{aligned}
$$

which proves that $f$ and $g$ are homomorphisms.

Since $(a, b) \in S_{\gamma}$ implies $(f(a), g(b))=k(a, b) \in T_{\delta}$, where $\delta=h(\gamma)$, we have $r f(a)=\delta=h(\gamma)=h p(a)$, namely, $r f=h p$. Similarly, $s g=h q$.

CoRollary 3. In Corollary $2, k$ is (1) one-to-one into, (2) onto or (3) one-to-one onto, respectively, if and only if there exists $h, f$ and $g$, all of which are (1) one-to-one into, (2) onto or (3) one-to-one onto, satisfying all the conditions in Corollary 2.

Proof. Sufficiency. It is easily proved by considering the mapping $k$ defined by $k(a, b)=(f(a), g(b))$, in each case.

Necessity (1) Let $k$ be one-to-one into. Then $k^{-1}\left(T_{\delta}\right)$ is rectangular if it is not empty, and so it is contained in only one $S_{\delta}$ by Corollary 1 to Theorem 1. Therefore $h$ is one-to-one into. Now it is easy to see that $f$ and $g$ are one-to-one.

(2) Let $k$ be onto. Then

$$
h(\Gamma)=h(u(S))=v k(S)=v(T)=\Delta,
$$

which shows that $h$ is onto. Now it is obvious that $f$ and $g$ are onto.

(3) Obvious by (1) and (2).

The case (3) of this corollary can be restated as follows.

CoRollary 4. The decomposition of a regular band into the spined product of a left regular band and a right regular band is unique up to idomorphism.

5. Characterizations by identities. Let $X=\{x, y, \cdots\}$ be a set whose elements we will call variables. A word is an element of the free semigroup $F=F(X)$. A pair of words $(P, Q)$ is called an identity and is written $P=Q$. 
Let $S$ be a band. Then we say $S$ satisfies an identity $P=Q$, if $f(P)=f(Q)$ for every homomorphism $f: F \rightarrow S$.

An identity $P=Q$ is said to imply an identity $P^{\prime}=Q^{\prime}$ if any band satisfying $P=Q$ also satisfies $P^{\prime}=Q^{\prime}$. Thus any identity implies the identity $x^{2}=x$, that is idempotence. If $P=Q$ implies $P^{\prime}=Q^{\prime}$ and $P^{\prime}=Q^{\prime}$ implies $P=Q$, then the identities are equivalent.

Let $X^{\prime}$ also be a set of variables. Let $t_{0}: X \rightarrow X^{\prime}$ be any transformation, then it induces a homomorphism $t: F(X) \rightarrow F\left(X^{\prime}\right)$ which coincides with $t_{0}$ on $X$.

It is easy to see that $P=Q$ implies $t(P)=t(Q)$.

Lemma 14. $P=Q$ implies $t(P)=t(Q)$ for any transformation of variables $t_{0}$.

The following lemmas are also straightforward.

Lemma 15. If $P=Q$ implies $P=P^{\prime}, Q=Q^{\prime}$, then $P=Q$ implies $P^{\prime}=$ $Q^{\prime}$.

Lemma 16. If $P=Q$ implies $P^{\prime}=Q^{\prime}$, then $P=Q$ implies both $P P^{\prime}=$ $Q Q^{\prime}$ and $P^{\prime} P=Q^{\prime} Q$.

REMARK 4. We can take the free idempotent semigroup generated by $X$ instead of the free semigroup generated by $X$. It makes no essential difference in the argument.

An identity $P=Q$ is said to be homotypical if both $P$ and $Q$ contain the same variables explicitly, otherwise it is said to be heterotypical. Thus an identity $x y=x$ is heterotypical, but an identity $x y=y x$ is homotypical.

If $P$ is a word $x_{1} x_{2} \cdots x_{n}$, then we call $x_{1}$ the head of $P$ and $x_{n}$ the tail of $P$.

THEOREM 5. An identity $P=Q$ is equivalent to left (right) singularity if and only if ${ }^{2}$

(1) $P=Q$ is heterotypical,

(2) $P$ and $Q$ have the same (different) heads,

(3) $P$ and $Q$ have different (the same) tails.

Proof. Sufficiency. Let $P=Q$ satisfy (1), (2) and (3) above. Then the words $P$ and $Q$ are expressed by $x \cdots x_{1}$ and $x \cdots x_{2}$, respectively, where $x_{1}$ is different from $x_{2}$, and either $x_{1}$ or $x_{2}$, but not both, may be the same as $x$. By assumption (1) either $P$ or $Q$ contains a variable $y$, which the other does not. Assume that $P$ contains $y$.

A transformation $X \rightarrow X$ defined by $y \rightarrow y$, all other variables $\rightarrow x$ sends the words $P, Q$ to $P^{\prime}, Q^{\prime}$ where $P^{\prime}$ is $x \cdots y \cdots x$ or $x \cdots y(\cdots$

\footnotetext{
${ }^{2}$ The "only if" part will be proved right after the proof of Lemma 17 below.
} 
stands for $x$ 's, $y$ 's or nothing) and $Q^{\prime}$ is $x^{n}$ for some positive integer $n$. Now any band satisfies the identities $P^{\prime}=x y x$ or $P^{\prime}=x y$, according as $P^{\prime}$ is $x \cdots y \cdots x$ or $x \cdots y$, and $Q^{\prime}=x$. Thus by using Lemmas 14 and 15 we have that $P=Q$ implies either (i) $x y x=x$ or (ii ) $x y=x$.

Since (i) is rectangularity, and rectangularity implies both $P=x x_{1}$ and $Q=x x_{2}$ by Lemma 2 , the identity $P=Q$ implies $x x_{1}=x x_{2}$ by Lemma 15. It is now easy to see that the identity $x x_{1}=x x_{2}$ implies left singularity, by a suitable transformation. (ii) itself shows left singularity.

Thus $P=Q$ implies left singularity.

Conversely, the identity $x y=x$ implies any identity of the form

$$
x \cdots y=x \cdots x \text { or } x \cdots y=x \cdots z,
$$

where $x, y, z$ are all different and $\cdots$ stand for any sequence of variables. Thus $x y=x$ implies any identity satisfying the conditions in the theorem.

THEOREM 6. An identity $P=Q$ is equivalent to rectangularity if and only $i f^{2}$

(1) $P=Q$ is heterotypical,

(2) $P$ and $Q$ have the same heads,

(3) $P$ and $Q$ have the same tails.

Proof. Let $P=Q$ be an identity satisfying (1), (2) and (3) above. Then we can assume that the word $P$ is $x \cdots y \cdots z$ and $Q$ is $x \cdots z$, where $Q$ does not contain the variable $y$, while $z$ can be the same as $x$. Now the transformation $y \rightarrow y$, all other variables $\rightarrow x$, implies the identity $x y x=x$, which is equivalent to rectangularity.

Conversely, rectangularity $x y x=x$ implies any identity of the form $x \cdots z=x \cdots z$ by Lemma 2 . Thus it implies any identity satisfying the above conditions.

REMARK 5. It is easily verified that all identities mentioned in Remark 2 satisfy the above three conditions.

Theorem 7. An identity $P=Q$ is equivalent to triviality, that is $x=y$, if and only if ${ }^{2}$

(1) $P=Q$ is heterotypical,

(2) $P$ and $Q$ have different heads,

(3) $P$ and $Q$ have different tails.

Proof. Let $P=Q$ be an identity satisfying the above condition. Then it implies both identities $z P=z Q$ and $P z=Q z$, where $z$ is a variable which is not contained in both $P$ and $Q$, by Lemma 16 . The former is equivalent to left singularity, while the latter is equivalent to right singularity by Theorem 5 . Thus $P=Q$ implies both left and right singu- 
larity. Hence it implies triviality.

Conversely, triviality implies any identity.

Lemma 17. Any semilattice satisfies any homotypical identity.

Proof. Let $P=Q$ be any homotypical identity whose variables are $x_{1}, \cdots, x_{n}$. Let $S$ be any semilattice. Then it is clear that $S$ satisfies the both identities, $P=x_{1} x_{2} \cdots x_{n}$ and $x_{1} x_{2} \cdots x_{n}=Q$. Thus $S$ satisfies the identity $P=Q$.

Proof of the necessity in Theorem 5, 6 and 7.

Let $P=Q$ be an identity which is equivalent to triviality, left (right) singularity or rectangularity. Let $S$ be the two-element semilattice. If $P=Q$ is homotypical, then $S$ satisfies this identity by the preceding lemma. But $S$ is not rectangular nor left (right) singular nor trivial. So this identity can not be homotypical. Thus it must be heterotypical. This takes care of the part (1) of the theorems.

Let $A(B)$ be the two-element left (right) singular band. Then $A(B)$ is neither right (left) singular nor trivial. Also $A(B)$ satisfies any identity $P=Q$ if the heads (tails) of $P$ and $Q$ are the same.

(i) Assume that $P=Q$ is equivalent to triviality. Then the heads (tails) of $P$ and $Q$ must be different. For, if not, $A(B)$ which is not trivial satisfies this identity. This proves the necessity of (2) and (3) in Theorem 7.

(ii) Assume that $P=Q$ is equivalent to left (right) singularity. Then the tails (heads) of $P$ and $Q$ must be different. For, if not, then $B(A)$, which is not left (right) singular, satisfies this identity. This takes care of (3) of Theorem 5 .

Now the heads (tails) of $P$ and $Q$ must be the same. For, if not, this identity is equivalent to triviality by Theorem 7 , which has already been proved completely. But there exists a left (right) singular band which is not trivial, for example, $A(B)$.

This takes care of (2) of Theorem 5 .

(iii) Assume that $P=Q$ is equivalent to rectangularity.

Then the heads of $P$ and $Q$ are the same and so are their tails. For, if not, the identity is equivalent to triviality or left singularity or right singularity by the preceding argument. Also there exists a band which is rectangular but neither left nor right singular nor trivial, for example, $A \times B$. This ends the proof of (2) and (3) in Theorem 6 .

Thus the classification of all heterotypical identities into four distinct cases is now completed.

THeorem 8. An identity $P=Q$ is equivalent to commutativity if it satisfies the following conditions: 
(1) $P=Q$ is homotypical,

(2) $P$ and $Q$ have different heads,

(3) $P$ and $Q$ have different tails.

Proof. Let $P=Q$ be an identity satisfying the above conditions (1), (2) and (3). Then we can assume the word $P$ is $x \cdots$, and $Q$ is $y \cdots$, $x \neq y$. Thus $P=Q$ implies $P x y=Q x y$. Now the transformation: $y \rightarrow y$, all other variables $\rightarrow x$ on the latter identity implies the identity $x y=$ $y x y$, which is equivalent to right regularity. Similarly, $P=Q$ implies left regularity. Thus by Lemma 10 the identity $P=Q$ implies commutativity.

Conversely, commutativity implies any homotypical identity.

Before stating the conditions for an identity to be equivalent to left or right regularity, we shall introduce the concept of initial and final parts, by which we can reduce both members of an identity to simpler forms.

If the word $P^{\prime}$, say $x_{i_{1}} x_{i_{2}} \cdots x_{i_{k}}$, is the word which is obtained by writing down all the distinct variables of the word $P$, say $x_{1} x_{2} \cdots x_{n}$, from the left, we call $P^{\prime}$ the initial part of $P$ and denote it by $q(P)$. Similarly, we can define the final part of $P, r(P)$, dually with respect to left and right. Thus if the word $P$ is $x y x z x$, then the initial part and the final part of $P$ are $x y z$ and $y z x$, respectively, that is $q(P)$ is $x y z$ and $r(P)$ is $y z x$.

When $P$ and $Q$ have the same initial (final) parts, we say that the identity $P=Q$ is coinitial (cofinal). Note that if an identity is coinitial or cofinal then it must be homotypical.

THEOREM 9. An identity $P=Q$ is equivalent to left (right) regularity, if it satisfies the following two conditions:

(1) $P=Q$ is coinitial (cofinal),

(2) $P$ and $Q$ have different tails (heads).

Proof. Let $P=Q$ satisfy the above two conditions. Then by (1) $P$ and $Q$ must have the same head, say $x$, By (2) one of $P$ or $Q$ has a tail which is different from $x$, say $P$ is $x \cdots y$, where $y \neq x$.

Let $t_{0}$ be the transformation defined by $y \rightarrow y$, all other variables $\rightarrow$ $x$. Then we have two identities $t_{0}(P)=x y$ and $t_{0}(Q)=x y x$. Thus we have left regularity, $x y=x y x$.

Conversely it is obvious that left regularity implies $P=P^{\prime}$ for any word $P$, where $P^{\prime}$ is the initial part of $P$. Thus left regularity implies any coinitial identity. Hence it implies any identity satisfying the above conditions.

The problem of finding the characteristic conditions for an identity 
on bands to be equivalent to regularity still remains open.

6. Free regular bands. By the free (left, right) regular band generated by a non-empty set $X$, we mean a band $S$ such that

(1) there exists a mapping $i: X \rightarrow S$, which is called the imbedding mapping,

(2) $i(X)$ generates $S$,

(3) $S$ is (left, right) regular,

(4) for any (left, right) regular band $T$ and for any mapping $j$ : $X \rightarrow T$, there exists a homomorphism $h: S \rightarrow T$ such that $j=h i$.

REMARK 6. In this definition, the imbedding mapping is not assumed to be one-to-one, but this property is proved easily in this case. Also it is easy to see that if there are two such free regular bands for a given set $X$, then they are isomorphic fixing every point of $X$ pointwise under the imbedding mappings. So if there exists a free (left, right) regular band, it is unique up to isomorphism. The homomorphism $h$ in (4) is also unique.

The free commutative band, i. e., the free semilattice generated by $X$ is defined similarly.

In this section we shall construct the free (left, right) regular band from a given set $X$.

Let $X$ be a non-empty set. Let $S$ be the set of all non-empty subsets of $X$ consisting of a finite number of points. Then we have the following

LEMMA 18. The above defined $S$ is the free semilattice generated by $X$ under the multiplication defined by $y z=y \cup z$, where $\cup$ denotes the union operation.

Proof. It is obvious that $S$ forms a semilattice generated by $\{\{x\}$ : $x \in X\}$. Let $i: X \rightarrow S$ be defined by $i(x)=\{x\}$. Let $T$ be a semilattice and $j: X \rightarrow T$ any mapping. Then the mapping $h: S \rightarrow T$ defined by $h(y)=j\left(x_{1}\right) j\left(x_{2}\right) \cdots j\left(x_{n}\right)$ where $y=\left\{x_{1}, x_{2}, \cdots, x_{n}\right\}$, is a homomorphism by commutativity and by idempotence, satisfying $j(x)=h(\{x\})=h(i(x))$, that is $j=h i$. Thus $S$ is the free semilattice generated by $X$.

Let $X$ be a non-empty set. Let $F=F(X)$ be a free semigroup generated by $X$. Then $F$ is the set of all finite sequences of points of $X$ with juxtaposition multiplication. We imbed $X$ in $F$ in the natural way under $k: X \rightarrow F$.

Consider the two mappings, the initial part $q: F \rightarrow F$ and the final part $r: F \rightarrow F$, defined in the preceding section. Let $A_{0}=q(F) \subset F, B_{0}=$ $r(F) \subset F$ be the images of $F$ under $q, r$. Note that not only are $q$ and 
$r$ not homomorphisms but also $A_{0}$ and $B_{0}$ can never be subsemigroups of $F$. To make them form bands we define other multiplications in $A_{0}$ and in $B_{0}$ as follows:

$$
\begin{aligned}
& m\left(a, a^{\prime}\right)=q\left(a a^{\prime}\right), \text { for } a, a^{\prime} \in A_{0}, \\
& n\left(b, b^{\prime}\right)=r\left(b b^{\prime}\right), \text { for } b, b^{\prime} \in B_{0} .
\end{aligned}
$$

Let $a, a^{\prime} a^{\prime \prime} \in A_{0}$. Then

$$
m\left(m\left(\alpha, a^{\prime}\right), \alpha^{\prime \prime}\right)=m\left(q\left(\alpha a^{\prime}\right), a^{\prime \prime}\right)=q\left(q\left(a \alpha^{\prime}\right) a^{\prime \prime}\right)=q\left(a a^{\prime} a^{\prime \prime}\right) .
$$

Similarly $m\left(a, m\left(a^{\prime}, a^{\prime \prime}\right)\right)=q\left(a a^{\prime} a^{\prime \prime}\right)$. Therefore $m$ is an associative multiplication on $A_{0}$.

Moreover $m(a, a)=q(a a)=q(a)=a$ and

$$
m\left(m\left(a, a^{\prime}\right), a\right)=q\left(a a^{\prime} a\right)=q\left(a a^{\prime}\right)=m\left(a, a^{\prime}\right) .
$$

Therefore $A_{0}$ forms a left regular band under the multiplication $m$. Similarly, $B_{0}$ is a right regular band under the multiplication $n$. We shall denote these bands by $A$ and $B$ instead of $A_{0}$ and $B_{0}$, because of the difference of multiplications.

It is now simple to see that $q: F \rightarrow A$ and $r: F \rightarrow B$ are both onto homomorphisms. Since $F$ is generated by $k(X), A$ and $B$ are generated by $i(X)$ and $j(X)$, respectively, where $i=q k$ and $j=r k$.

Let $A^{\prime}$ be any left regular band and $i^{\prime}: X \rightarrow A^{\prime}$ any mapping. Since $F$ is the free semigroup generated by $X$, there exists a homomorphism $f: F \rightarrow A^{\prime}$ such that $i^{\prime}=f k$. For any $w \in F$ we have $f(w)=$ $f(q(w))$, because $A^{\prime}$ is left regular. Thus there exists a homomorphism $h: A \rightarrow A^{\prime}$ such that $f=h q$. Therefore

$$
i^{\prime}=f k=(h q) k=h(q k)=h i .
$$

Hence $A$ is the free left regular band generated by $X$. Similarly $B$ is the free right regular band generated by $X$.

Consider the free semilattice $\Gamma$ generated by $X$ with its imbedding $c: X \rightarrow \Gamma$ (Lemma 18). Then since $\Gamma$ is both left and right regular, there exist homomorphisms $s: A \rightarrow \Gamma$ and $t: B \rightarrow \Gamma$ such that $s i=c=t j$. It is obvious that

$$
s(a)=\left\{x_{1}, x_{2}, \cdots, x_{n}\right\} \text {, if } a=i\left(x_{1}\right) i\left(x_{2}\right) \cdots i\left(x_{n}\right) .
$$

Let $A_{\gamma}=s^{-1}(\gamma)$ and $B_{\gamma}=t^{-1}(\gamma)$ for $\gamma \in \Gamma$. Then it is easy to see that $A_{\gamma}\left(B_{\gamma}\right)$ is left (right) singular. Thus by Corollary 3 to Theorem 1, $A \sim$ $\sum\left\{A_{\gamma}: \gamma \in \Gamma\right\}$ and $B \sim \sum\left\{B_{\gamma}: \gamma \in \Gamma\right\}$ are the structure decomposition of $A$ and $B$. Thus we have the following

THEOREM 10. Let $X$ be a non-empty set. Let $\Gamma$ be the free semilattice obtained in Lemma 18. Let $A(B)$ be the set of all linearly ordered 
non-empty finite subsets of $X$ together with the multiplication defined by juxtaposition deleting all second letters which appear in the expression of the juxtaposition product reading from the left (right). Let $s: A \rightarrow \Gamma(t$ : $B \rightarrow \Gamma$ ) be the mapping defined by $s(a)(t(b))=$ the set of all distinct points contained in a (b). Let $A_{\gamma}=s^{-1}(\gamma)$ and $B_{\gamma}=t^{-1}(\gamma)$ for $\gamma \in \Gamma$. Then $A(B)$ is the free left (right) regular band generated by $X$ and $A \sim \sum\left\{A_{\gamma}: \gamma \in \Gamma\right\}$ $\left(B \sim \sum\left\{B_{\gamma}: r \in \Gamma\right\}\right)$ is its structure decomposition.

COROLLARY. The free left (right) regular band generated by $n$ elements consists of $\sum_{i=1}^{n}\left(\begin{array}{l}n \\ i\end{array}\right) i !=n ! \sum_{i=0}^{n-1} 1 / i !$ elements.

Proof. Each $A_{\gamma}$ consists of $i$ ! elements when $\gamma$ contains $i$ elements, since $A_{\gamma}$ consists of all permutations of points of $\gamma$.

Let $A(B)$ and $\Gamma$ be the free left (right) regular band and the free semilattice generated by $X$ with the imbedding mappings $i: X \rightarrow A(j:$ $X \rightarrow B$ ) and $c: X \rightarrow \Gamma$, respectively. Since $\Gamma$ is both left and right regular, there exist homomorphisms $s: A \rightarrow \Gamma$ and $t: B \rightarrow \Gamma$ such that $s i=c=t j$.

Let $C$ be the spined product of $A$ and $B$ with respect to $\Gamma$ with $s$ and $t$ as spine homomorphisms. Then $C$ is the subset of $A \times B$ consisting of elements $(a, b)$ such that $s(a)=t(b)$. Now since $s(i(x))=(s i)(x)=$ $c(x)=(t j)(x)=t(j(x))$ the element $(i(x), j(x))$ is in $C$. Define $k: X \rightarrow C$ by $k(x)=(i(x), j(x))$.

Now we shall prove that $k(X)$ generates $C$.

Pick any element $(a, b) \in C$. Then $s(a)=t(b) \in \Gamma$. Since $A$ and $B$ are generated by $X$, we have

$$
a=i\left(x_{1}\right) i\left(x_{2}\right) \cdots i\left(x_{m}\right), \quad b=j\left(y_{1}\right) j\left(y_{2}\right) \cdots j\left(y_{n}\right) .
$$

Thus

$$
c\left(x_{1}\right) c\left(x_{2}\right) \cdots c\left(x_{m}\right)=s(a)=t(b)=c\left(y_{1}\right) c\left(y_{2}\right) \cdots c\left(y_{n}\right) .
$$

Therefore the subset consisting of the points $x_{1}, x_{2}, \cdots, x_{m}$ coincides with that consisting of the points $y_{1}, y_{2}, \cdots, y_{n}$.

Since $A$ is left regular we have

$$
i\left(x_{1}\right) i\left(x_{2}\right) \cdots i\left(x_{m}\right) i\left(y_{1}\right) i\left(y_{2}\right) \cdots i\left(y_{n}\right)=i\left(x_{1}\right) i\left(x_{2}\right) \cdots i\left(x_{m}\right)=a .
$$

Similarly,

$$
j\left(x_{1}\right) j\left(x_{2}\right) \cdots j\left(x_{m}\right) j\left(y_{1}\right) j\left(y_{2}\right) \cdots j\left(y_{n}\right)=j\left(y_{1}\right) j\left(y_{2}\right) \cdots j\left(y_{n}\right)=b .
$$

Thus we have $(a, b)=k\left(x_{1}\right) k\left(x_{2}\right) \cdots k\left(x_{m}\right) k\left(y_{1}\right) k\left(y_{2}\right) \cdots k\left(y_{n}\right)$, which proves that $k(X)$ generates $C$.

Next, we shall prove that $C$ is the free regular band generated by $X$. 
Let $C^{\prime}$ be any regular band and $k^{\prime}: X \rightarrow C^{\prime}$ any mapping. Since $C^{\prime}$ is regular, it is the spined product of a left regular band $A^{\prime}$ and a right regular band $B^{\prime}$ with respect to a semilattice $\Gamma^{\prime}$, where $s^{\prime}: A^{\prime} \rightarrow$ $\Gamma^{\prime}$ and $t^{\prime}: B^{\prime} \rightarrow \Gamma^{\prime}$ are the spine homomorphisms. Now the freeness of $A, B$ and $\Gamma$ implies the existence of homomorphisms $f: A \rightarrow A^{\prime}, g: B \rightarrow$ $B^{\prime}$ and $h: \Gamma \rightarrow \Gamma^{\prime}$ such that

$$
f i=u^{\prime} k^{\prime}, \quad g j=v^{\prime} k^{\prime}, \quad h c=d^{\prime} k^{\prime},
$$

where $u^{\prime}: C^{\prime} \rightarrow A^{\prime}, v^{\prime}: C^{\prime} \rightarrow B^{\prime}$ are natural and $d^{\prime}: C^{\prime} \rightarrow \Gamma^{\prime}$ are such that

$$
S^{\prime} u^{\prime}=d^{\prime}=t^{\prime} v^{\prime}
$$

Let $u: C \rightarrow A, v: C \rightarrow B$ be natural and $d: C \rightarrow \Gamma$ be such that $s u=d=t v$. Take $(a, b) \in C$. Then $s(a)=t(b)$ by definition. Since $C$ is generated by $k(X)$, there exist $x_{1}, x_{2}, \cdots, x_{n} \in X$. such that

$$
a=i\left(x_{1}\right) i\left(x_{2}\right) \cdots i\left(x_{n}\right), b=j\left(x_{1}\right) j\left(x_{2}\right) \cdots j\left(x_{n}\right) .
$$

Put $a^{\prime}=f(a)$ and $b^{\prime}=g(b)$. Then by (1) and (2) we have

$$
\begin{aligned}
& s^{\prime}\left(a^{\prime}\right)=s^{\prime} f(a)=\prod_{\nu=1}^{n} s^{\prime} f\left(x_{\nu}\right)=\prod_{\nu=1}^{n} s^{\prime} u^{\prime} k^{\prime}\left(x_{\nu}\right)=\prod_{\nu=1}^{n} d^{\prime} k^{\prime}\left(x_{\nu}\right), \\
& t^{\prime}\left(b^{\prime}\right)=t^{\prime} g(b)=\prod_{\nu=1}^{n} t^{\prime} g j\left(x_{\nu}\right)=\prod_{\nu=1}^{n} t^{\prime} v^{\prime} k^{\prime}\left(x_{\nu}\right)=\prod_{\nu=1}^{n} d^{\prime} k^{\prime}\left(x_{\nu}\right) .
\end{aligned}
$$

Thus $s^{\prime}\left(a^{\prime}\right)=t^{\prime}\left(b^{\prime}\right)$. Therefore $\left(a^{\prime}, b^{\prime}\right) \in C^{\prime}$, that is $(f(a), g(b)) \in C^{\prime}$. Hence there exists a mapping $p: C \rightarrow C^{\prime}$ defined by $p(a, b)=(f(a), g(b))$.

It is now easy to see that $p$ is a homomorphism. Moreover for $x \in$ $X$ we have by (1)

$$
k^{\prime}(x)=\left(u^{\prime} k^{\prime}(x), v^{\prime} k^{\prime}(x)\right)=(f i(x), g j(x))=p k(x),
$$

because $k(x)=(i(x), j(x))$, and accordingly $k^{\prime}=p k$. This completes the proof that $C$ is the free regular band generated by $X$. Thus we have the following

Theorem 11. Let $X$ be a non-empty set. Let $A, B$ and $\Gamma$ be the free left regular, the free right regular and the free commutative band generated by $X$, respectively, so that $\Gamma$ is regarded as the structure semilattice of both $A$ and $B$. Then the free regular band generated by $X$ is the spined product of $A$ and $B$ with respect to $\Gamma$.

CoROLlaRY. The regular band generated by $n$ distinct elements consists of

$$
\sum_{i=1}^{n}\left(\begin{array}{l}
n \\
i
\end{array}\right)(i !)^{2}=n ! \sum_{i=1}^{n} \frac{i !}{(n-i) !}
$$


elements.

Proof. Each $A_{\gamma} \times B_{\gamma}$ consists of $(i !)^{2}$ elements when contains $i$ elements.

\section{REFERENCES}

1. A. H. Clifford, Bands of semigroups, Proc. Amer. Math. Soc. 5 (1954), 499-504.

2. Naoki Kimura, On semigroups, Dissertation, Tulane University (1957), 1-133.

3. David McLean, Idempotent semigroups, Amer. Math. Monthly 61 (1954), 110-113.

TUlane UNIVERSITY AND TOKYO INSTITUTE OF TEChNOLOGY 



\section{PACIFIC JOURNAL OF MATHEMATICS}

\section{EDITORS}

\section{H. L. Royden}

Stanford University

Stanford, California

\section{R. A. Beaumont}

University of Washington

Seattle 5 , Washington

\author{
A. L. Whiteman
}

University of Southern California

Los Angeles 7, California

E. G. Straus

University of California

Los Angeles 24, California

\section{ASSOCIATE EDITORS}
E. F. BECKENBACH
A. HORN
L. NACHBIN
G. SZEKERES
C. E. BURGESS
V. GANAPATHY IYER
I. NIVEN
F. WOLF
M. HALL
R. D. JAMES
T. G. OSTROM
E. HEWITT
M. S. KNEBELMAN
M. M. SCHIFFER
K. YOSIDA

\section{SUPPORTING INSTITUTIONS}

\author{
UNIVERSITY OF BRITISH COLUMBIA \\ CALIFORNIA INSTITUTE OF TECHNOLOGY \\ UNIVERSITY OF CALIFORNIA \\ MONTANA STATE UNIVERSITY \\ UNIVERSITY OF NEVADA \\ OREGON STATE COLLEGE \\ UNIVERSITY OF OREGON \\ UNIVERSITY OF SOUTHERN CALIFORNIA
}

\author{
STANFORD UNIVERSITY \\ UNIVERSITY OF UTAH \\ WASHINGTON STATE COLLEGE \\ UNIVERSITY OF WASHINGTON \\ * * * * \\ AMERICAN MATHEMATICAL SOCIETY \\ CALIFORNIA RESEARCH CORPORATION \\ HUGHES AIRCRAFT COMPANY \\ THE RAMO-WOOLDRIDGE CORPORATION
}

Mathematical papers intended for publication in the Pacific Journal of Mathematics should be typewritten (double spaced), and the author should keep a complete copy. Manuscripts may be sent to any of the editors. All other communications to the editors should be addressed to the managing editor, E. G. Straus at the University of California, Los Angeles 24, California.

50 reprints per author of each article are furnished free of charge; additional copies may be obtained at cost in multiples of 50 .

The Pacific Journal of Mathematics is published quarterly, in March, June, September, and December. The price per volume (4 numbers) is $\$ 12.00$; single issues, $\$ 3.50$. Back numbers are available. Special price to individual faculty members of supporting institutions and to individual members of the American Mathematical Society: $\$ 4.00$ per volume; single issues, $\$ 1.25$.

Subscriptions, orders for back numbers, and changes of address should be sent to Pacific Journal of Mathematics, 2120 Oxford Street, Berkeley 4, California.

Printed at Kokusai Bunken Insatsusha (International Academic Printing Co., I.td.), No. 10, 1-chome, Fujimi-cho, Chiyoda-ku, Tokyo, Japan.

PUBLISHED BY PACIFIC JOURNAL OF MATHEMATICS, A NON-PROFIT CORPORATION

The Supporting Institutions listed above contribute to the cost of publication of this Journal, but they are not owners or publishers and have no responsibility for its content or policies. 


\section{Pacific Journal of Mathematics}

\section{Vol. 8, No. 2 \\ April, 1958}

John Herbert Barrett, Second order complex differential equations with a real independent variable ............................ 187

Avner Friedman, Remarks on the maximum principle for parabolic equations and its applications ......................... 201

Richard Robinson Goldberg, An inversion of the Stieltjes transform ....... 213

Olavi Hellman, On the periodicity of the solution of a certain nonlinear integral equation .................................. 219

Gilbert Helmberg, A theorem on equidistribution on compact groups...... 227

Lloyd Kenneth Jackson, Subfunctions and the Dirichlet problem ......... 243

Naoki Kimura, The structure of idempotent semigroups. I ............ 257

Stephen Kulik, A method of approximating the complex roots of equations........................................ 277

Ancel Clyde Mewborn, A note on a paper of L. Guttman.............. 283

Zeev Nehari, On the principal frequency of a membrane ............ 285

G. Pólya and I. J. Schoenberg, Remarks on de la Vallée Poussin means and convex conformal maps of the circle ...................... 295

B. M. Stewart, Asymmetry of a plane convex set with respect to its centroid .......................................... 335

Hans F. Weinberger, Lower bounds for higher eigenvalues by finite difference methods

Edwin Weiss and Neal Zierler, Locally compact division rings ......... 369

Bertram Yood, Homomorphisms on normed algebras ................. 373 